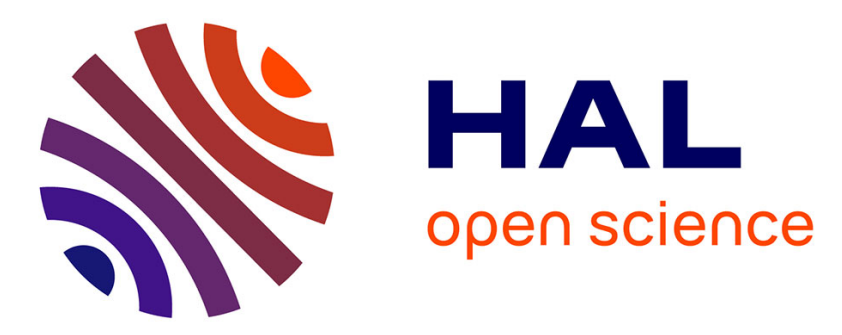

\title{
Hommes-femmes, l'introuvable égalité. La place contradictoire des femmes dans la société française
}

Alain Bihr, Roland Pfefferkorn

\section{To cite this version:}

Alain Bihr, Roland Pfefferkorn. Hommes-femmes, l'introuvable égalité. La place contradictoire des femmes dans la société française. Recherches et prévisions, 2000, 61, pp.19-33. 10.3406/caf.2000.904 . hal-01292151

\section{HAL Id: hal-01292151 \\ https://hal.science/hal-01292151}

Submitted on 22 Mar 2016

HAL is a multi-disciplinary open access archive for the deposit and dissemination of scientific research documents, whether they are published or not. The documents may come from teaching and research institutions in France or abroad, or from public or private research centers.
L'archive ouverte pluridisciplinaire HAL, est destinée au dépôt et à la diffusion de documents scientifiques de niveau recherche, publiés ou non, émanant des établissements d'enseignement et de recherche français ou étrangers, des laboratoires publics ou privés. 
Hommes-femmes, l'introuvable égalité. La place contradictoire des femmes dans la société française

Alain Bihr, Roland Pfefferkorn

Citer ce document / Cite this document :

Bihr Alain, Pfefferkorn Roland. Hommes-femmes, l'introuvable égalité. La place contradictoire des femmes dans la société française. In: Recherches et Prévisions, n61, 2000. pp. 19-33.

doi : 10.3406/caf.2000.904

http://www.persee.fr/doc/caf_1149-1590_2000_num_61_1_904

Document généré le 05/01/2016 


\title{
Hommes-femmes, l'introuvable égalité
}

\author{
La place contradictoire des femmes dans la société française
}

\author{
Alain Bihr * et Roland Pfefferkorn **
}

La situation des femmes au sein de la société française est fondamentalement contradictoire. Au terme de quatre décennies de bouleversements importants, les acquis sont réels, mais restent fragiles. Les limites doivent d'emblée être soulignées en raison notamment du succès d'une rhétorique convenue sur la "féminisation des moeurs " ou sur l'émancipation des femmes qui serait " désormais réalisée ", en raison aussi de l'accent mis tout au long des débats de ces dernières années sur la parité et sur les inégalités dans la représentation politique. Le brouillage idéologique empêche trop souvent de prendre l'exacte mesure des inégalités persistantes entre les sexes. C'est pourquoi on tentera de brosser, certes à gros traits, un tableau de la place contradictoire des femmes dans la société française d'aujourd'hui, en mettant l'accent plus particulièrement sur l'espace domestique qui reste l'épicentre de la domination masculine.

* Laboratoire cultures et sociétés en Europe(UPRESA 7043) et LIO (Laboratoire d'intelligencedes organisations). Maître de conférences en sociologieà l'universitédeHaute-Alsace. ** Laboratoire cultures et sociétés en Europe (UPRESA 7043). Professeur agrégé de sciences sociales à l'université Marc-Bloch de Strasbourg.

Le titre de cet article reprend en partie celui de l'ouvrage... l'école, au travail, au sein du couple, de la famille ou encore dans l'espace public, les femmes occupent une position qui reste, le plus souvent, subalterne. L'infériorisation sociale des femmes peut se vérifier à différents niveaux, même si, pour l'essentiel depuis les années soixante, les rapports entre hommes et femmes se sont largement modifiés. Dans le sens d'une plus grande égalité entre hommes et femmes, cette transformation des rapports sociaux de sexes est, cependant, loin d'être achevée. L'examen détaillé de l'évolution récente permet précisément de nuancer l'ampleur de ces transformations et, surtout, d'en préciser le sens contradictoire.

En premier lieu, on peut observer des transformations au sein des rapports inégalitaires traditionnels entrehommes et femmes. Dans les pratiques et les structures qui les engendrent et les maintiennent, les écarts entre hommes et femmes se sont sensiblement réduits. Ainsi, les femmes ont pu avancer sur la voie de leur émancipation comme sujets à la fois personnels et collectif. On pense en particulier à l'accès aux études et à une activité professionnelle salariée.

En deuxième lieu, malgré les apparences, des rapports inégalitaires perdurent, quelquefois simplement déplacés ou transformés. Largement souligné pour la vie publique, et plus particulièrement pour la vie politique, ce phénomène est tout aussi marqué dans l'espace domestique, aspect peut-être sous-estimé ces dernières années.

Enfin, de nouvelles formes d'inégalités sont apparues, nées de la collision entre les inégalités traditionnelles maintenues etles transformations a priori positives survenues au sein des rapports entre hommes et femmes. Elles sont autant d'effets pervers de ces transformations par ailleurs libératrices. 
Incontestablement, un certain nombre d'inégalités traditionnelles entre hommes et femmes se sont réduites et, par conséquent, la situation des femmes dans la société française contemporaine s' est relativement améliorée. Pour l'essentiel, cette évolution positive s'est produite sousl'action conjointe de trois facteurs : le développement de la scolarisation des filles, l'entrée massive des femmes dans l'activité professionnelle et la maîtrise de la fécondité.

\section{Une meilleure scolarisation des filles}

Au cours des dernières décennies, les filles ont non seulement rattrapé mais encore dépassé les garçons sur le plan scolaire: durée moyenne des études, niveau moyen de diplômes, taux de redoublement ou de retard scolaire, taux de réussite aux examens, niveau moyen aux épreuves de contrôle des acquis scolaires, etc. Cela se vérifie dans l'enseignement primaire et secondaire, de même que dans les deux premiers cycles du supérieur. Il faut attendre le troisième cycle du supérieur (et encore, dans certaines filières seulement) pour observer le maintien de la traditionnelle supériorité masculine (Baudelot et Establet, 1992 ; Blöss et Frickey, 1987).

Ces transformations des années soixantequatre-vingt-dix avaient été précédées par un lent rattrapage des filles pendant la période précédente. Dans l'entre-deuxguerres (1920-1940), alors que la plupart des jeunes ne fréquentent pas l'école audelà du primaire, la scolarisation des garçons dépasse nettement celle des filles. Cependant, les écarts varient d'une catégorie sociale à l'autre. L'écart entre les sexes est particulièrement marqué dans le salariat ouvrier et chez les indépendants.

Après la Seconde Guerre mondiale, dans les années cinquante, on enregistre une prolongation de la scolarité à l'issue du primaire dans tous les milieux sociaux avec un rythme toutefois différencié. Les filles rattrapent d'abord leur retard parmi les enfants de cadres supérieurs et moyens ; plus tard le phénomène s'étend aux autres catégories.

Au milieu des années soixante, la suppression del'examen d'entréeen sixièmea permis d'ouvrir plus largement l'enseignement secondaire aux enfants des milieux populaires. Dès lors, les écarts entre sexes se creusent, mais cette fois au détriment des garçons. Cesécarts sontd'autant plusimportants que l'on descend les degrés de l'échelle sociale. L'écart entre sexes est le plus réduit parmi les enfants des catégories favorisées qui poursuivent très majoritairement leurs études (tableau 1).

Le résultat de la progression de la scolarisation féminine est spectaculaire. En 1993, $37 \%$ des femmes de nationalité française âgées de 25 ans à 39 ans sont titulaires d'un diplôme égal ou supérieur au baccalauréat, contre seulement $30 \%$ des hommes. Dans la génération précédente, les 50-59 ans, les hommes dépassaient encore les femmes ( $21 \%$ contre $19 \%$ ).

\section{Une réussite scolaire supérieure à celle des garçons}

En 1994, globalement, toutes filières et tous niveaux confondus, $48 \%$ des filles de 16 ans à 25 ans étaient scolarisées contre un peu plus de $43 \%$ des garçons. Quelles que soient les classes d'âge retenues, les filles sont aujourd'hui plus scolarisées que les garçons, mais l'écart tend à se réduire avec l'avancée en âge et la prolongation des études. Depuis quelques décennies, on observe, dans toutes les catégories sociales, une réussite scolaire des filles supérieure à celle des garçons, et ce de la maternelle au baccalauréat. Quel que soit l'âge d'entrée en sixième, les filles accèdent plus souvent en classe de seconde, puis en terminale, que les garçons. De plus, leur taux de réussite est supérieur, aussi bien pour les bacs généraux que pour les bacs technologiques. Les écarts entre les sexes sont cependant moins importants en haut de l'échelle sociale : $86 \%$ des filles de cadres entrées en sixième en 1980 se retrouvent en terminale, contre $81 \%$ des garçons (soit cinq points 
Tableau 1 - Accès en terminale des cohortes entrées en sixième en 1973 et 1980

\begin{tabular}{|c|c|c|c|c|c|c|c|c|c|c|c|c|c|c|}
\hline \multirow{2}{*}{$\begin{array}{l}\text { Cohorte } \\
\text { Age scolaire }\end{array}$} & \multicolumn{6}{|c|}{1973} & \multicolumn{6}{|c|}{1980} & \multirow{2}{*}{\multicolumn{2}{|c|}{$\begin{array}{c}\begin{array}{c}\text { Nombre } \\
\text { d'accédantes }\end{array} \\
\begin{array}{c}\text { Pour } 100 \\
\text { accédants }\end{array}\end{array}$}} \\
\hline & \multicolumn{2}{|c|}{ A l'heure } & \multicolumn{2}{|c|}{ En retard } & \multicolumn{2}{|c|}{ Ensemble } & \multicolumn{2}{|c|}{ A l'heure } & \multicolumn{2}{|c|}{ En retard } & \multicolumn{2}{|c|}{ Ensemble } & & \\
\hline Sexe & G & $\mathbf{F}$ & G & $\mathbf{F}$ & G & $F$ & G & $\mathbf{F}$ & G & $\mathbf{F}$ & $G$ & $F$ & 73 & 80 \\
\hline $\begin{array}{l}C S P \text { : } \\
\text { favorisées }\end{array}$ & 69 & 80 & 19 & 26 & 57 & 70 & 78 & 84 & 24 & 25 & 69 & 77 & 123 & 112 \\
\hline moyennes & 47 & 61 & 7 & 14 & 28 & 42 & 52 & 65 & 10 & 15 & 38 & 52 & 150 & 134 \\
\hline défavorisées & 34 & 50 & 4 & 8 & 14 & 25 & 43 & 54 & 8 & 9 & 25 & 35 & 179 & 140 \\
\hline Total & 50 & 63 & 7 & 11 & 28 & 40 & 58 & 67 & 11 & 12 & 41 & 51 & 143 & 124 \\
\hline
\end{tabular}

Source : Enquêtes de suivis 1973 et 1980 (DEP/MEN). Jean-Pierre Terrail, Destins scolaires de sexe, Population, 1992, $\mathrm{n}^{\circ} 3$.

Sexe $: G=$ garçons $; F=$ filles. CSP : catégories socioprofessionnelles. Favorisées $:$ industriels, gros commerçants, cadres supérieurs, professions libérales, enseignants, cadres moyens. Moyennes : employés, contremaîtres, artisans, petits commerçants, agriculteurs. Défavorisées : ouvriers, personnels de service, salariés agricoles.

Lecture du tableau (douze premières colonnes) : $69 \%$ des fils des catégories favorisées entrés à l'heure en sixième ont accédé en terminale, et $19 \%$ y sont entrés en retard ; les deux dernières colonnes se lisent ainsi : pour 100 fils des catégories favorisées entrés en sixième en 1973, 123 filles sont dans ce cas.

d'écart); $35 \%$ des filles des catégories " défavorisées » contre $25 \%$ des garçons (soit dix points d'écart).

Les sociologues et les spécialistes des sciences de l'éducation ont tenté de donner des explications cohérentes de cette progression de la scolarisation féminine depuis le début des années soixante. Les explications centrées sur la mobilisation des principales intéressées et de leurs familles, notamment des mères, semblent les plus convaincantes. Elles ne nient pas la réalité de la domination qui pèse sur les femmes, mais elles soulignent qu'ellen'est pas une fatalité et qu'elle peut être combattue.

Ainsi, Jean-Pierre Terrail met l'accent sur l'investissement plus actif dans l'appropriation du savoir qui permet aux jeunes filles, et en particulier à celles d'origine populaire, d'échapper aux déterminants ordinaires de l'échec scolaire. Il insiste sur le rôle joué dans cette dynamique émancipatrice par la mobilisation familiale, et en premier lieu celle des mères, apportant à la fois un encouragement quotidien et une caution symbolique à la réussite scolaire des filles. Plus encore que le rapport des mères à l'emploi, c'est leur rapport à l'école et au savoir qui jouerait un rôle déterminant dans leur implication dans la scolarité de leurs enfants. Les relations mère-fille particulièrement étroites, faites de dialogue et de complicité, renforceraient la confiance en la capacité de réussir des filles. Les mères des milieux populaires seraient ainsi amenées à encourager chez leurs filles l'ambition de ce qu'elles n'ont pas pu réaliser pleinement elles-mêmes (Terrail, 1992 a, 1992 b, 1992 c et 1992/1993).

\section{Le rôle essentiel des mères}

A partir d'une enquête approfondie menée dans la banlieue de Lyon, Bernard Lahire souligne également, dans les milieux populaires, le rôle essentiel du modèle des mères (voire des grands-mères, des sœurs, des tantes ou des voisines) qui entretiennent un rapport régulier et étroit avec l'écrit. Ces mères, en effet, tiennent non seulement l'écriture domestique, mais elles se chargent aussi de la plupart des démarches de caractère administratif (Lahire, 1995). Une profonde dynamique émancipatoire serait ainsi à l'œuvre depuis une trentaine d'années. Elle résulterait de l'activité ordinaire des individus, et plus particulièrement de celle des femmes (Terrail, 1995).

Ces dernières décennies auront également vu se réduire considérablement les diffé- 
rences de taux d'activité entre hommes et femmes. On aura notamment assisté à une entrée massive des femmes dans le salariat. Depuis le début des années soixante, les femmes ont véritablement investi le monde du travail (tableau 2). Le nombre d'hommes actifs est resté relativement stable, autour de quatorze millions, mais le nombre de femmes actives est passé de sept millions à près de douze millions. Même si on tient compte des seuls actifs ayant un emploi, les chiffres sont tout aussi parlants. De 1962 à 1999, le nombre des hommes occupant un emploi a légèrement reculé tandis que le nombre de femmes occupant un emploi progresse de près de quatre millions.

Depuis le début de la crise, le mouvement ne s'est pas ralenti en dépit de la difficulté grandissante à trouver un emploi. En 1997, près de $80 \%$ des femmes âgées de 25 ans à 49 ans sont désormais actives contre à peine $50 \%$ il y a un peu moins de vingt ans. Parmilesjeunes générations (25ans à 39 ans), les femmes sont actives à plus de $90 \%$ quand elles sont sans enfants, à plus de $80 \%$ avec deux enfants, et à près de $55 \%$ lorsqu'elles sont mères de trois enfants et plus.

Toutes ces transformations s'effectuent de surcroît dans un contexte de forte augmentation de la part des salarié(e)s dans la population active. En 1994, 83,5\% des hommes et $89 \%$ des femmes occupant un emploi sont des salariés. Les professions indépendantes et les agriculteurs exploitants ne représentent plus qu'une part très faible des actives et des actifs. Globalement, environ un tiers seulement des non-salariés sont des femmes. Cependant, il faut préciser que près de $95 \%$ des hommes non salariés sont réellement des indépendants, avec ou sans salariés, contre seulement $60 \%$ des femmes. Les autres femmes non salariées sont en fait des « aides familiales » qui participent au travail de l'entreprise agricole, artisanale ou commerciale de leur conjoint sans pour autant être associées à sa direction. L'indépendance de ces indépendantes est donc pour le moins limitée. Enfin, parmi l'ensemble des salariés, la part des femmes a très fortement progressé ces dernières années, puisqu'elle est passée d'un peu plus de $41 \%$ en 1982 à près de $46 \%$ en 1994.

\section{Le travail féminin : un phénomène social massif}

Le modèle dela femme au foyer a été délaissé par la très grande majorité des femmes. Il y a évidemment une synergie forte entre ces deux premiers facteurs. La perspective $\mathrm{d}^{\prime}$ occuper un emploi, avec ce qu'il signifie d'autonomie personnelle, a été une puissante motivation d'investissement scolaire. Inversement, l'obtention de diplômes aura permis aux femmes d'accéder à l'emploi salarié qualifié, y compris dans les postes d'encadrement. Cependant, hommes et femmes sont encore loin d'occuper les mêmes types d'emplois et d'entretenir le même rapport à l'emploi (Maruani, 1998 ; Maruani et Nicole-Drancourt, 1989 a).

Le travail féminin est devenu un phénomène social massif. Il est maintenant fortement inscrit dans la réalité sociale et tout semble

Tableau 2 - Population active par sexe de 1962 à 1999 (en milliers)

\begin{tabular}{|l|l|r|r|r|r|r|r|c|}
\hline & Sexe & $\mathbf{1 9 6 2}$ & $\mathbf{1 9 6 8}$ & $\mathbf{1 9 7 5}$ & $\mathbf{1 9 8 2}$ & $\mathbf{1 9 9 0}$ & $\mathbf{1 9 9 5}$ & $\mathbf{1 9 9 9}$ \\
\cline { 2 - 8 } Population active & Homme & 13158 & 13559 & 13907 & 14192 & 14212 & 13911 & 14175 \\
& Femme & 6585 & 7124 & 8132 & 9585 & 11051 & 11368 & 11808 \\
Population active & Homme & 13046 & 13321 & 13533 & 13258 & 13037 & 12551 & 12723 \\
occupée & Femme & 6489 & 6925 & 7675 & 8460 & 9426 & 9793 & 10200 \\
Chômeurs & & & & & & & & \\
& Homme & 112 & 238 & 374 & 934 & 1175 & 1360 & 1452 \\
& Femme & 96 & 199 & 457 & 1125 & 1625 & 1575 & 1608 \\
\hline
\end{tabular}

Source : Données sociales, 1993 (INSEE) ; INSEE-Première, juin 1995, n³89; INSEE-Première, juin 1999, nº 658. 
indiquer qu'il est devenu irréversible. Le travail des femmes participe désormais à la construction del'identité féminine : les filles, à l'école, se préparent à un métier comme les garçons, et celles qui ne cherchent pas d'emploi à l'issue de leur scolarité sont très rares.

Cet investissement accru des femmes dans le monde du travail est également lié à deux autres phénomènes : la diffusion de la contraception et leur plus grande autonomie à l'égard des rapports conjugaux. Trente-trois ans après la loi Neuwirth (1967), plus de deux femmes sur trois (entre 20 ans et 44 ans) utilisent une méthode contraceptive. Parmi ces dernières, près des deux tiers utilisent la pilule et un quart le stérilet. Aujourd'hui, seule une petite minorité de femmes $(3 \%)$ encoure le risque d'une grossesse non désirée. Cette maîtrise par les femmes de leur fécondité leur aura permis de limiter les naissances (en fait, le mouvement en ce sens est amorcé depuis deux bons siècles) et surtout de maîtriser le calendrier des naissances. Ces conditions sont essentielles pour l'entrée comme pour le maintien des femmes dans le salariat.

\section{Une plus grande autonomie à l'égard des rapports conjugaux}

L'ensemble des mouvements précédents - meilleure formation et qualification professionnelle, entrée massive dans le salariat, maîtrise de leur fécondité - ont permis aux femmes de conquérir une plus grande autonomieà l'égard des rapports conjugaux. Elles ont été pleinement actrices des transformations qui ont affecté les relations conjugales au cours de ces vingt dernières années : développement de la cohabitation hors mariage, développement du divorce aussi, développement du célibat surtout parmi les femmes les plus dotées scolairement et professionnellement, même si, pour ce dernier point, il convient peut-être de nuancer le propos en raison des règles de fonctionnement du marché matrimonial qui dressent des obstacles spécifiques sur la voie de la mise en couple de ces femmes.
Inversement, on a assisté au maintien d'un certain nombre d'inégalités traditionnelles entre hommes et femmes, entraînant la stagnation de la situation des femmes. On peut repérer deux points forts de résistance dela domination masculine. D'abord au sein de l'espace public où les hommes continuent à accaparer l'essentiel des positions de pouvoir. Les débats autour de la parité politique ont souligné la place inégale des femmes dans l'espace politique. Mais les inégalités traditionnelles subsistent largement au sein de l'espace privé dont les tâches continuent à être assurées prioritairement par les femmes. On développera essentiellement ce second aspect dans la mesure où le débat sur la "parité domestique » se fait largement attendre.

\section{La résistance de la domination masculine}

Le premier pôle de résistance de la domination masculine se situe au sein de l'espace public. L'antique monopolisation par les hommes de cet espace s'est maintenu jusqu'à présent. On peut s'en tenir à deux chiffres. A l'Assemblée nationale, en 1997, à peine $10 \%$ de femmes siègent et moins de $6 \%$ au sein du Sénat qui vient d'être renouvelé. Le Parlement français est très loin de la situation atteinte dans les pays nordiques (38,9\% de femmes parlementaires et même $42,7 \%$ en Suède) et fait à peine mieux que celui de la République islamique d'Iran... L'essentiel se jouera, à l'avenir, dans la mise en œuvre effective de la parité désormais inscrite dans la Constitution (Sineau, 1988).

Mais cette domination masculine se vérifie plus largement dans la monopolisation $d u$ pouvoir dans d'autres lieux : au sommet des grandes entreprises, dans les organisations syndicales ou les administrations publiques, dont les fonctions de responsabilités restent accaparées, dans leur immense majorité, par des hommes. En outre, l'espace public ne se réduit pas à la seule scène du pouvoir. Il comprend aussi ce qu'on pourrait appeler la scène mondaine : cet espace où les corps 
des hommes et des femmes se donnent à voir les uns aux autres. L'analyse des discours publicitaires, de la mode, des magazines masculins et féminins, de certaines pratiques (comme les seins nus sur les plages) montrent que cette scène mondaine reste dominée par les hommes : le corps féminin reste pour l'essentiel un objet de contemplation et de convoitise pour l'œil masculin (Goffman, 1977; Kaufmann, 1995).

\section{Les tâches domestiques: toujours assurées par les femmes}

En dépit des évolutions relevées supra, les femmes continuent à assurer la plus grande partie du travail domestique. En 1999, elles consacrent presque autant de temps aux activités domestiques (hors bricolage et jardinage) qu'en 1986: trois heures trentetrois minutes par jour en moyenne pour une active occupée et quatre heures vingtcinq minutes pour une inactive. En treize ans, les femmes actives occupées auront enregistré une baisse de quatre minutes et les hommes, dans la même situation, une hausse de six minutes.

Les variations sont infimes et le temps passé aux activités domestiques est pour l'essentiel resté stable. Les hommes consacrent désormais une heure quinze minutes en moyenne par jour aux tâches domestiques au lieu d'une heure neuf minutes. Les femmes actives occupées consacrent toujours près de trois fois plus de temps à ces activités que leurs homologues masculins (Brousse, 2000 ; Dumontier et Pan Ke Shon, 1999). L'activité domestique inclut un ensemble d'activités très varié, allant des tâches ménagères proprement dites à la gestion des revenus et du patrimoine de la famille en passant par l'éducation des enfants et par l'organisation del'espace-temps familial.Ces activités représentent une somme de travail énorme, qui dépasse en importance celle mesurée annuellement par le PIB. Elle est pourtant longtemps restée invisible. La manière dontces activités sont réparties entre les conjoints permet de déterminer ce qu'il en est aujourd'hui du pouvoir au sein de l'espace conjugal et familial.
Par tâches ménagères, on entend les tâches d'entretien matériel des membres de la famille, de son logement et de son équipement. Elles constituent l'aspect le plus visible parce que le plus important du travail domestique. La mécanisation et la socialisation partielles des tâches domestiques ont fait disparaître les plus astreignantes, les plus sales et les plus dangereuses. En ce sens, leur charge quotidienne de travail domestique s'est incontestablement allégée, condition de leur entrée dans une activité professionnelle.

Que l'on pense simplement combien le branchement des foyers sur les réseaux d'eau courante et d'eaux usées, de gaz et d'électricité, les a dispensées des corvées d'eau, de bois, d'entretien des feux, d'évacuation des ordures et des excréments. Que l'on pense aussi combien des appareils électroménagers tels que le lave-linge hier, le lave-vaisselle aujourd'hui les dispensent de longues et pénibles tâches quotidiennes, de surcroît peu gratifiantes, ou les réduisent considérablement. Le recul de l'auto-production alimentaire et vestimentaire, grâce à l'augmentation générale du niveau de vie, leur a de même permis de gagner du temps, sinon pour elles, du moins pour s'engager dans une activité professionnelle. Enfin, le développement de la restauration hors domicile (sur les lieux de travail ou d'enseignement) a produit des effets analogues.

\section{Une répartition très inégale des tâches ménagères}

Mais en dépit de ces progrès incontestables de la condition féminine dans l'espacetemps de la vie privée, et comme le montrent les enquêtes Emploi du temps réalisées par l'INSEE, c'est encore aux femmes qu'incombe l'essentiel de ces tâches ménagères d'entretien (Brousse, 2000 ;Dumontier et Pan Ke Shon, 1999 ; Haicault, 1984).

Les tâches ménagères ne demeurent pas seulement inégalement réparties quant à leur durée, elles le sont aussi quant à leur nature. On a pu distinguer trois grandes catégories de tâches (Zarca, 1990). Les «tâ- 
ches féminines ", prises en charge quasi exclusivement par les femmes, comprennent ce qui concerne le linge (lavage, repassage, raccommodage) mais aussi le nettoyage des sanitaires; les hommes ne les prennent en charge que dans moins de $5 \%$ des cas, même s'ils peuvent y participer à titre secondaire dans $10 \%$ à $20 \%$ des cas.

Les " tâches masculines ", prises en charge très majoritairement par les hommes, comprennent essentiellement le portage $\mathrm{du}$ combustible (bois, charbon, mazout), l'entretien de l'automobile (lavage, réparation) et le bricolage; les femmes prennent, cependant, plus souvent ces tâches masculines en charge (dans $10 \%$ à $20 \%$ des cas) que les hommes ne prennent en charge les tâches féminines.

Quant aux "tâches négociables", qui " continuent d'être prises en charge par une majoritédefemmes, mais (auxquelles) plus d'un tiers des hommes, et parfois même plus des deux tiers (...) participent 》 (Zarca, 1990), elles regroupent notamment les courses, la cuisine, la vaisselle, le ménage, etc. ; " ces tâches peuvent être effectuées à égalité dans une proportion non négligeable de cas : jusqu'à $10,6 \%$ pour les courses ", alors que ce n'est le cas que dans une infime minorité $(2 \%$ à $3 \%$ ) en ce qui concerne les tâches masculines ou féminines. Ces résultats sont quasi identiques à ceux obtenus douze ans auparavant lors d'une enquête du même type : seules certaines tâches " négociables " (la vaisselle, les courses, la cuisine) semblent avoir été un peu plus prises en charge par les hommes.

\section{L'image conventionnelle des attributs et vertus}

Cette division repose en fait sur quelques oppositions pertinentes qui véhiculent une image très conventionnelle des attributs et vertus censésêtre spécifiquement masculins ou féminins. Aux femmes les travaux " légers " (ne requérant pas la force physique), supposant en revanche la maîtrise de savoir-faire traditionnels, essentiellement préoccupés de la propreté (du lieu, des vêtements). Aux hommes les travaux de force, ou supposant la maîtrise d'objets et d'instruments techniques plus modernes, plutôt tournés vers l'extérieur.

Tandis que les tâches féminines sont plutôt de l'ordre de la reproduction, impliquant la répétition périodique (quotidienne, hebdomadaire, mensuelle, saisonnière) des mêmes gestes et des mêmes opérations, avec ce qu'elles impliquent de routine et de sentiment d'ennui et même de vacuité, véritable travail de Sisyphe toujours à reprendre, les tâches masculines sont davantage del'ordre de la production, voire de la création, suscitant la fierté de soi et la reconnaissance d'autrui. Le résultat d'un bricolage réussi est plus visible et se laisse plus admirer qu'un tas de linge repassé ou même un ménagebien fait. C'est au contraire lorsqu'il n'est pas fait ou mal fait qu'un «ménage » se voit...

\section{Un partage différent selon les milieux sociaux}

Evidemment, selon les milieux socioculturels, la division des tâches ménagères est plus ou moins accentuée. Le pourcentage $\mathrm{d}$ 'hommes effectuant une "tâche féminine » ou une " tâche négociable » est variable d'une catégorie socioprofessionnelle à l'autre. C'est parmi les indépendants (agriculteurs, artisans, commerçants et chefs d'entreprise, professions libérales) qu'il est le plus faible et parmi les cadres supérieurs qu'il est le plus élevé. Seul $1 \%$ des premiers prend en charge des tâches féminines, alors que c'est le cas de $5,8 \%$ des seconds. Si à peine un sur dix $(9,4 \%)$ des premiers consent à effectuer des "tâches négociables", ils sont proportionnellement trois fois plus nombreux dans ce cas $(30,3 \%)$ parmi les seconds.

On ne s'étonnera pas non plus, dans ce cas, de constater que l'intensité de cette division varie également en fonction du niveau de formation des conjoints. Plus il est élevé, plus les hommes acceptent de participer aux tâches "féminines » ou "négociables", sans doute parce que le niveau d'exigence 
en matière d'égalité dans le partage des tâches domestiques est plus élevé parmi les femmes diplômées et que ces dernières se retrouvent dans des milieux sociaux plus aisés avec un recours aux employés de maison. En moyenne, les femmes salariées obtiennent plus fréquemment la prise des tâches "féminines » ou " négociables » par leur conjoint que les femmes inactives.

En étudiant la gestion du linge dans un ensemble de jeunes couples partageant l'idéologie progressiste del'égalité des sexes dans tous les domaines, Jean-Claude Kaufmann a mis en évidence le processus de reproduction en leur sein de la division traditionnelle des tâches ménagères (Kaufmann, 1992). Dans un premier temps, sous l'emprise de l'exigence égalitaire, ces tâches sont accomplies à tour de rôle par les deux conjoints. Rapidement, cependant, un autre partage intervient, chacun des conjoints délimitant un « territoire personnel ", un ensemble de tâches ménagères dont il prend la charge : à l'un la cuisine, à l'autre le ménage, etc. Le passage d'un partage à l'autre s'accomplit sous la pression de l'inégale disponibilité des conjoints selon les différents moments de la journée ou de la semaine, mais aussi, et surtout, selon des inégalités de compétence et plus encore $d$ 'exigence de chacun en matière de tâches ménagères, héritées de leur histoire réciproque.

Or ces héritages ne sont pas les mêmes pour un homme ou une femme. Par son éducation, unefemme est généralement plus apte à accomplir la plupart des tâches ménagères qu'un homme, tandis que l'accomplissement de ces tâches lui paraît simultanément plus important ou plus urgent qu'à son conjoint. En conséquence, elle aura plus tendance à se charger de ces tâches, aussi bien parce qu'il lui en coûte moins de les accomplir que parce qu'elle les jugera plus nécessaires mais aussi mieux accomplies par elle. L'homme s'en déchargera pour des raisons inverses des précédentes. Ainsi s'insinue progressivement une division inégalitaire des tâches domestiques, dérive qui induit un conflit permanent, peu compatibleavec lesentiment amoureux qui est à la base du lien conjugal et familial.

\section{La prise en charge des enfants : un autre facteur d'inégalité}

La prise en charge des enfants est un autre facteur d'inégalité. On entend par prise en charge non seulement les soins donnés aux nourrissons, mais la garde des enfants, la surveillance de leurs devoirs et leçons quand ils sont scolarisés, la participation à leurs jeuxintérieurs et extérieurs, les promenades et sorties avec eux, etc. Les pères consacrent en moyenne à peine une vingtaine de minutes à leurs enfants par jour, les mères actives s'en occupent pendant près d'une heure et les mères inactives plus de deux heures.

Cette inégalité se confirme lorsqu'on détaille les différentes tâches qu'implique cette prise en charge. La garde des enfants en bas âge non scolarisés, et non pris en charge par une structure collective ou un tiers, incombe encore quasi exclusivement aux femmes. De même, lorsque les enfants sont malades, ce sont les mères qui les gardent. Cette situation les conduit fréquemment à rogner sur leurs jours de congé comme si, une fois de plus, l'activité professionnelle de la femme et les enjeux qu'elle représente restaient en définitive secondaires au regard de sa mission maternelle. Témoignage de cet état de fait, un projet de loi élaboré par le Secrétariat d'Etat aux droits des femmes, fixant un nombre de jours de congés non transférables accordés à chacun des deux époux pour garder les enfants malades, non abouti. Les mères se chargent également, pour la plus grande partie, de l'aide au travail scolaire de leurs enfants : elles y passent en moyenne deux fois plus de temps que les pères. De même, ce sont les mères qui, beaucoup plus fréquemment que les pères, prennent contact avec les enseignants. Il existe, cependant, d'importantes différences entre les milieux sociaux: chez les ouvriers et les agriculteurs, le père rencontre le moins souvent les enseignants. 
L'investissement inégal des pères et des mères dans l'éducation de leurs enfants persiste donc très largement. Une des questions centrales qui se pose est de " déterminer la place réciproque des fonctions parentales et d'éducation et celle des personnes sexuées qui sont appelées à tenir ces fonctions, exemplairement les parents »(Neyrand, 1999). Rien ne permet d'attribuer au père $a$ priori une fonction symbolique universelle indépassable qui justifierait sa moindre implication dans l'éducation de ses enfants, voire dans les tâches domestiques dans leur ensemble.

\section{La fonction paternelle est fragilisée}

Cependant, comme le remarque Gérard Neyrand, "la définition d'une fonction de soin par son rapport à la figure de la mère et sa désignation commefonction maternelle, demême que la définition d'une fonction d'autorité, par son rapport à la figure du père et sa désignation comme fonction paternelle, figent la représentation de la parentalité et de l'éducation dans des attributions à chacun des sexes qui amènent logiquement les parents à "incarner" ces fonctions » (Neyrand, 1999).

L'évolution contemporaine dela conjugalité, avec le développement de l'union libre et l'augmentation considérable de la part des naissances hors mariage, contribue aussi à fragiliser l'exercice de la fonction paternelle. En effet, comme le remarque Françoise Hurstel: "Le statut légal du père dans le concubinage participe d'une certaine vision anachronique de la paternité et de la maternité, celle d'un père absent, ne prenant pas ses responsabilités, fuyant, et d'une mère indispensable et seul parent fiable pourl'enfant ». L'auteur se demande, par conséquent, si "l'autorité parentale » accordée d'emblée à la mère ne vient pas renforcer un fantasme transmis de mère en fille et de mère en fils. "Sur le versant de la collectivité il peut être formulé par "les enfants sont aux mères", "les mères savent mieux pour les enfants", et sur le versant de la subjectivité c'est le fantasme de la "toute-puissance maternelle" lié à la relation primordiale qui unit tout enfant à sa mère "
(Hurstel, 1996 b). Le droit ne vient-il pas avaliser un fantasme individuel et une croyance collective qui tendent à exclure symboliquement (et réellement) le père et quijustifierait in fine sa moindre implication dans l'éducation de ses enfants?

\section{Au sein du couple, l'homme est en position dominante}

Aujourd'hui majoritaire au sein des couples, la structure du pouvoir apparaît peu égalitaire. L'homme reste en position dominante. Certes, il partage le plus souvent la fonction de direction avec sa conjointe. Cependant, il continue à soustraire du champ de compétence de cette "direction collégiale » les décisions concernant son activité professionnelle propre, alors qu'à l'inverse, dans la majorité des cas, il continue à prendre part aux décisions concernantl'activité professionnelle desonépouse. Les grosses dépenses seraient engagées après discussion collective, les arguments du mari l'emportant, cependant, le plus souvent. L'épouse gérerait plutôt les petites dépenses au quotidien (nourriture, services, vêtements, loisirs, etc.). Surtout, l'homme parvient visiblement à se décharger sur son épouse d'une bonne partie des activités d'intendance et d'entretien. Autrement dit, si la direction est désormais le plus souvent collégiale, l'intendance et la "production » restent encore essentiellement l'affaire des femmes. C'est bien en cela qu'elles continuent à être dominées (Glaude et De Singly, 1986).

Différents facteurs sont susceptibles de favoriser un rééquilibrage du pouvoir domestique en faveur des femmes. Au premier rang figure l'entrée dans le salariat. Un bon diplôme, qui va souvent de pair avec une position favorable danslahiérarchiesocioprofessionnelle,estégalement un facteur susceptible de favoriser ce rééquilibrage. Inversement, la présence d'enfants semble favoriser le maintien ou l'introduction d'un partage inégal du pouvoir. On comprend aussi la défiance de plus en plus grande de certaines jeunes femmes à l'égard de l'entrée prématurée et sans condition dans les rapports conjugaux et familiaux. 
En fait, ce n'est pas seulement parce que les tâches et rôles dont sont traditionnellement chargées les femmes sont peu valorisants que les hommes répugnent à les assumer. L'enjeu d'un rééquilibrage total dans la division du travail domestique est bien plus fondamental. Il y va de l'identité même des hommes mais aussi de celle des femmes, identité qui s'est précisément définie dans et par des modèles familiaux fondés sur cette division traditionnelle. Les acteurs du couple contemporain sont traversés par la contradiction entre leur exigence d'égalité, qui plaide en faveur de l'abolition de la division sexuelle du travail domestique, et leur identité personnelle, reposant précisément en partie sur cette division. Ce conflit marque plus particulièrement les femmes.

\section{Entre exigence d'égalité et identité personnelle}

Quoi qu'il en soit, la division sexuelle du travail domestique est bien le centre de toutes les inégalités entre hommes et femmes. Couplée avec la maternité dont elle renforce considérablement le poids, cette division fait obstacle sinon à l'entrée et au maintien des femmes dans le salariat, du moins à un investissement professionnel équivalent de celui des hommes, et partant, à des carrières professionnelles aussi prestigieuses ou tout simplement continues (Desplanques et Saboulin, 1986). Les possibilités d'ascension sociale des femmes dans et par leur travail se trouvent mises en cause.

Plus fondamentalement encore, en maintenant des rapports inégalitaires au sein de l'espace familial où se façonne très tôtl'identité sexuelle des individus, cette division renforce des modèles inégalement valorisés et valorisants del'homme et de la femme. Ces modèles conduisent non seulement les jeunes filles à intégrer très tôt l'idée qu'en dépit de tout, l'essentiel des tâches domestiques - et notamment les moins prestigieuses - leur reviendra en lot conjugal, mais encore à adapter en conséquence leurs ambitions scolaires et professionnelles. C'est bien en définitive à l'intérieur des rapports conjugaux et familiaux, sous couvert de l'amour, que continue toujours à se reproduire l'inégalité fondamentale entre hommes et femmes.

Les deux ensembles de mouvements contradictoires esquissés dans les deux précédentes parties, avancées d'un côté, stagnation de l'autre, ne sont pas sans effets pervers. D'autant plus que la dynamique socio-économique d'ensemble des années 1980-1990 aggrave la situation.

\section{Un taux de chômage et de précarité supérieur à celui des hommes}

Si le taux d'activité des femmes n'a cessé de progresser au cours de ces vingt dernières années, dans un contexte de dégradation générale de la situation de l'emploi, c'est aussi au prix d'un taux de chômage et de précarité des femmes bien supérieur à celui des hommes. L'augmentation de leur activité est due en très grande partie à la progression de ces fameuses «formes particulières d'emplois » (c'est-à-direles différentes formes de travail précaire et de sous-emploi) : travail à domicile, travail au noir, travail intérimaire, contrats à durée déterminée, "emplois aidés » de diverses natures, sans compter le travail à temps partiel qui s'adresse massivement aux femmes. Autrement dit, les femmes plus que les hommes, ont été les victimes de la déréglementation néo-libérale du rapport salarial (Bouffartigue et Pendaries, 1990 ; Hirata et Senotier, 1996).

On peut, en particulier, incriminer le développement du travail à temps partiel (Bouffartigues, de Conink et Pendaries, 1992 ; Galtier, 1999 ; INSEE, 1995 ; Maruani et Nicole-Drancourt, $1989 \mathrm{~b}$ ). L'ensemble de ces "formes particulières d'emplois ", y compris le travail à temps partiel, représente aujourd'hui plus de cinq millions d'emplois contre deux millions en 1982. La montée rapide du travail à temps partiel est le signeavant-coureur d'une remise en cause de la dynamique de l'emploi des femmes. 
En effet, depuis 1980, la progression de l'emploi féminin correspond pour $80 \%$ à une augmentation du recours au travail à temps partiel. Tous les emplois nouveaux créés dans le secteur tertiaire destinés aux employés - très majoritairement des femmes - sont des emplois à temps partiel. Le travail à temps partiel doit donc être considéré, comme l'ont souligné à juste titre Margaret Maruani et Chantal NicoleDrancourt, comme "l'ennemi principal de l'égalité devant l'emploi».

\section{L'essor du travail \\ à temps partiel : une demande sociale des femmes?}

Ce développement du travail à temps partiel résulte avant tout des politiques de gestion de la main-d'œuvre des entreprises et des incitations gouvernementales. Pour justifier ces dernières, on invoque souvent une prétendue " demande sociale des femmes » pour concilier travail et famille. Le temps partiel concerne d'abord les femmes de moins de 25 ans, qui ne sont pas celles qui sont les plus écrasées par les charges domestiques, et les plus de 50 ans, qui en sont pour l'essentiel dégagées.

Sans vouloir dénier toute réalité à cette fameuse " demande sociale des femmes ", il convient surtout de souligner combien elle est contrainte dans le cas des femmes, en raison de leur prise en charge du travail domestique et des évolutions du marché du travail antérieurement évoquées. Le temps partiel contribue en fin de compte, et cela de manière décisive, à une redéfinition du statut du travail et de l'emploi, sur la base d'un critère de sexe. L'insertion professionnelle des jeunes filles est de plus en plus difficile et contraste avec leur meilleure réussite scolaire (Rack, 1998).

Parmi les mesures publiques dont les effets sont très problématiques pour les femmes, on peut également mentionner la mise en place de l'allocation parentale d'éducation, suivie en 1994 de son extension au deuxième enfant. Cette prestation constitue de facto une mesure de politique de l'emploi bien qu'étant présentée comme une mesure de politique familiale. Elle contribue, en effet, à retirer $\mathrm{du}$ marché du travail un nombre non négligeable de femmes pour qui la reprise d'activité ne sera pas aisée (Bonnet et Labbé, 1999 ; Fagnani, 1995, 1996 et 1998).

\section{Les conséquences des séparations sont plus lourdes pour les femmes}

On a rappelé plus haut que les femmes avaient pris une part active au processus de " libéralisation » des relations conjugales. Mais elles sont aussi les principales victimes de ce processus, notamment quand on envisage les conséquences inégales de la séparation. Elles sont toujours plus lourdes pour les femmes que pour les hommes. Parmi les facteurs explicatifs, la garde des enfants leur est confiée neuf fois sur dix, ce qui a pour conséquence une dégradation de leur situation matérielle et financière, mais aussi de leur situation relationnelle (notamment quant à la possibilité de reformer un couple).

On voit là comment une avancée sur un plan (la libéralisation des relations conjugales), couplée avec le maintien des relations inégalitaires (l'assignation des femmes à l'ordre domestique, en l'occurrence à la garde des enfants), se traduit en définitive par une dégradation ou du moins une fragilisation de la situation des femmes. Cependant, la garde des enfants n'a pas seulement des conséquences négatives. Une minoritécroissante de pères éprouve un profond sentiment d'injustice lorsque les enfants sont confiés à la mère, et certains d'entre eux s'estiment lésés.

Si seulement $20 \%$ des pères demandent la garde de leurs enfants en cas de divorce, seuls $20 \%$ d'entre eux obtiennent cette garde. Cette situation est parfois vécue d'autant plus douloureusement que certaines mères, qui ont encouragé leur conjoint à s'investir activement dans l'éducation de leurs enfants, revendiquent et obtiennent la garde au moment du divorce en s'appuyant sur le rôle traditionnel des mères. Dans ces 
situations de conflit conjugal, les femmes ne sont donc pas toujours en situation de « victimes ». Si le «pouvoir masculin » est incontestablement assorti de beaucoup de privilèges, il comporte néanmoins quelques revers (Hurstel, 1996 a et 1996 b ; Neyrand, 1999).

Cette dialectique d'invariance et de changement dont on a tenté de rendre compte brièvement résulte en premier lieu de la dynamique générale de la société. Mais on a aussi reconnu dans les changements enregistrés l'œuvre des volontés et des pratiques d'un nombre grandissant de femmes. En contribuant à l'ébranlement des rapports patriarcaux, elles ont su revendiquer et conquérir une autonomie nouvelle, sans pouvoir ni vouloir toujours s'émanciper totalement de ces rapports et maîtriser les contraintes nouvelles nées des transformations induites par leurs propres revendications et mouvements.

\section{L'émancipation féminine : une œuvre inachevée}

L'ambivalence d'un grand nombre de femmes à l'égard de ces rapports et du " modèle masculin » reste, dans la plupart des domaines, particulièrement frappante. Sujétion maintenue ou renouvelée dans ses formes, mais aussi autonomie et recherche d'autonomie grandissantes, tels sont finalement les deux pôles entre lesquels s'inscrit désormais, et provisoirement, l'existence des femmes dans la société française.

Les progrès accomplis sur la voie d'une égalité entre hommes et femmes ont un caractère limité, et les incertitudes quant à l'avenir des acquis récents en ce domaine restent importantes. On constate, par exemple, que le mouvement de réduction des écarts de salaires entre hommes et femmes observé entre le début des années soixante etlemilieu desannées quatre-vingtestbloqué depuis une bonne dizaine d'années et que, parallèlement, les pressions cantonnant les femmes au temps partiel s'accentuent de plus en plus (Maruani, 1998 ; Silvera, 1994/ 1995).
L'émancipation féminine reste une œuvre inachevée, à poursuivre, en prenant appui sur les acquis qui ont permis aux femmes de devenir, partiellement au moins, actrices de leur propre destin comme de celui de la société entière. Sur cette voie, le principal obstacle demeure probablement la perpétuation de la division inégalitaire des tâches et des fonctions au sein du couple et de la famille. Elle assigne encore en priorité les femmes à l'univers domestique. Dès lors que l'on cherche les raisons de la perpétuation du statut d'infériorité de la femme, $c^{\prime}$ est toujours à cet obstacle que l'on se heurte. D'ailleurs, symptomatiquement, avec la mixité de la représentation politique, l'aspect des rapports hommes/femmes a le moins bougé au cours des trois dernières décennies.

\section{L'univers domestique, « machine " à générer l'inégalité}

Pour l'ensemble de ces raisons, l'hypothèse $d^{\prime}$ 'une assignation des femmes à l'univers domestique constitue le noyau dur de la domination masculine contemporaine. S'attaquer directement à ce noyau paraît $a$ priori particulièrement difficile. $D^{\prime}$ une part, cela implique de s'en prendre à la vie privée qui relève de la liberté des individus et, d'autre part, c'est surtout remettre en question les identités sexuelles actuellement existantes, tant féminines que masculines. Cequi constituerait une véritable révolution culturelle, bien sûr souhaitable dans l'absolu, mais probablement très difficile à mettre en œuvre. En effet, il faudrait instaurer d'autres institutions domestiques et politiques, $d$ 'autres modes de socialisation des individus, un nouvel imaginaire social, etc.

Aussi, plutôtqu'une attaque frontale contre ce noyau dur ne vaudrait-il pas mieux développer une série d'attaques latérales? Si cette " machine » à générer et entretenir l'inégalité entre les sexes qu'est l'univers domestique a pu être en partie enrayée au cours des dernières décennies, c'est dans l'exacte mesure où les femmes ont pu s'y soustraire, essentiellement par la prolonga- 
tion de leur scolarité et par l'accès au travail salarié. D'où l'importance stratégique de tout ce qui peut continuer à détacher davantage encore les femmes à l'emprise de l'univers conjugal et familial. Il faut donc défendre et développer la scolarisation des filles et l'emploi des femmes. Il faut également renforcer les effets émancipateurs en favorisant leur accès à l'espace public et leur participation à la vie publique. En somme, il s'agit d'exacerber les contradictions qui définissent aujourd'hui la condition féminine selon ces trois axes : la scolarisation, l'accès et le maintien dans l'emploi (notamment salarié) et la prise de responsabilités dans l'espace public. 


\section{Références bibliographiques}

Aubin C. et Gisserot H., Les femmes en France : 1985-1995, Collection des rapports officiels, Paris, La Documentation française, 1994.

Barrère-Maurisson M.-A. (sous la dir.), Le sexe du travail. Structures familiales et système productif, Presses universitaires de Grenoble, 1984.

Baudelot C. et Establet R., Allez les filles !, Paris, Seuil, 1992.

Bihr A. et Pfefferkorn R., Hommes/Femmes, l'introuvable égalité. Ecole, travail, couple, espace public, Paris, Les éditions de l'Atelier, 1996.

Bihr A. et Pfefferkorn R., Déchiffrer les inégalités, Paris, Syros, 2e édition, 1999.

Blöss T. et Frickey A., Entre innovations et inégalités sociales : la scolarisation des femmes, Société française, 1987, $\mathrm{n}^{\circ} 24$.

Blöss T., Les rapports sociaux entre les sexes : permanences et changement, Problèmes politiques et sociaux, Paris, La Documentation française, 1994, $\mathrm{n}^{\circ} 732$.

Bonnet C. et Labbé M., L'activité des femmes après la naissance du premier et du deuxième enfant. L'impact de l'allocation parentale d'éducation, collection Etudes et statistiques, DREES, octobre 1999, n 10.

Bouffartigue P. et Pendaries J.-R., Activité féminine et précarisation de l'emploi, Travail et emploi, $\mathrm{n}^{\circ} 46,1992$.

Bouffartigue P., De Conink F. et Pendaries J.-R., Le nouvel âge de l'emploi à temps partiel. Un rôle nouveau lors des débuts de vie active des femmes, Sociologie du travail, XXXIII, octobre-décembre 1992, $\mathrm{n}^{\circ} 4$.

Bourdieu P., La domination masculine, Actes de la recherche en sciences sociales, septembre $1990, \mathrm{n}^{\circ} 84$.

Brousse C., La répartition du travail domestique entre conjoints : permanences et évolution de 1986 à 1999, France Portrait social, INSEE, édition 2000.

Cahiers du Mage, "Différences de sexe sur le marché du travail », IRESCO, 1995, n² 2 et 3-4.

Chabaud-Rychter D., Fougeyrollas-Schwebel D. et Sonthonnax F., Espace et temps du travail domestique, Paris, Méridiens Klincksieck, 1985.

Commaille J., Les stratégies des femmes, Paris, La Découverte, 1993.

De Singly F., L'amour, un bien privé, un mal public ?, Revue française des affaires sociales, $1988, \mathrm{n}^{\circ} 2$.

De Singly F. (sous la dir.), La famille : l'état des savoirs, Paris, La Découverte, 1991.

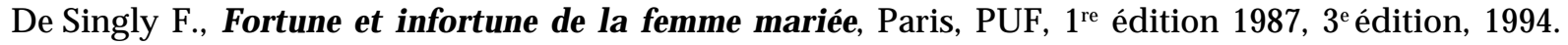

De Singly F., Les habits neufs de la domination masculine, Esprit, novembre 1993.

Desplanques G. et De Saboulin M.,Activitéféminine : carrières continues et discontinues, Economie et Statistique, novembre-décembre 1986, n 193-194.

Dumontier F. et Pan Ke Shon J.-L., En 13 ans, moins de temps contraint et plus de loisirs, INSEE-Première, octobre $1999, \mathrm{n}^{\circ} 675$.

Ephesia, La place des femmes. Les enjeux de l'identité et de l'égalité au regard des sciences sociales, Paris, La Découverte, 1995.

Fagnani J., L'allocation parentale d'éducation : effets pervers et ambiguïtés d'une prestation, Droit social, mars 1995, $\mathrm{n}^{\circ} 3$.

Fagnani J., Retravailler après une longue interruption. Le cas des mères ayant bénéficié de l'allocation parentale d'éducation, Revue française des affaires sociales, 1996, $\mathrm{n}^{\circ} 3$.

Fagnani J., Lacunes, contradictions et incohérences des mesures de conciliation travail/famille. Brefbilan critique, Droit social, juin $1998, \mathrm{n}^{\circ} 6$.

Fougeyrollas-Schwebel D., Travail domestique, services domestique, in Le monde du travail (sous la dir. de Kergoat J., Boutet J., Jacot H. et Linhart D.), Paris, La Découverte, 1998. 
Galtier B., Les temps partiels : entre emplois choisis et emplois " faute de mieux », Economie et statistiques, 1999, $\mathrm{n}^{\circ} 321-322,1 / 2$.

Glaude M. et De Singly F., L'organisation domestique : pouvoir et négociation, Economie et statistique, avril 1986, $\mathrm{n}^{\circ} 187$.

Goffman E., La ritualisation de la féminité, Actes de la recherche en sciences sociales, avril 1977, $\mathrm{n}^{\circ} 14$.

Haicault M., La gestion ordinaire de la vie en deux, Sociologie du travail, 1984, $\mathrm{n}^{\circ} 3$.

Hirata H. et Senotier D., Femmes et partage du travail, Paris, Syros, 1996.

Hurstel F., La déchirure paternelle, Paris, PUF, 1996 a.

Hurstel F., Paradoxes et fragilités de la paternité : les pères dans l'union libre, Revue des sciences sociales de la France de l'Est, Strasbourg, 1996 b.

INSEE, Données sociales, Paris, éditions 1990, 1993, 1996, 1999.

INSEE, Le travail à temps partiel, Liaisons sociales, DARES, collection Les dossiers thématiques, juillet 1995.

INSEE, Les familles monoparentales, collection Contours et caractères, Paris, 1994.

INSEE, Les femmes, collection Contours et caractères, Paris, $1^{\text {re }}$ édition 1991, $2^{\text {e }}$ édition 1995.

Kaufmann J.-C., Corps de femmes, regards d'hommes. Sociologie des seins nus, Paris, Nathan, 1995.

Kaufmann J.-C., La trame conjugale. Analyse du couple par son linge, Paris, Nathan, 1992.

Kergoat D., La division du travail entre les sexes in Le monde du travail (sous la dir. de Kergoat J., J. Boutet, Jacot H. et Linhart D. dir.), Paris, RESSY, La Découverte, 1998.

Lahire B., Tableaux de familles. Heurs et malheurs scolaires en milieu populaire, Paris, Gallimard et Seuil, 1995.

Langevin A., «Les effets du salariat féminin sur la socialisation des jeunes (approche comparative frèresoeur) ", CNRS, rapport de recherche pour la CNAF, 1989.

Laufer J., La féminité neutralisée, les femmes cadres dans l'entreprise, Paris, Flammarion, 1982.

Maruani M. (sous la dir.), Les nouvelles frontières de l'inégalité. Hommes et femmes sur le marché du travail, Paris, La Découverte, 1998.

Maruani M. et Nicole-Drancourt C., Au labeur des dames. Métiers masculins, emplois féminins, Paris, Syros, 1989 a.

Maruani M. et Nicole-Drancourt C., La flexibilité à temps partiel, Paris, La Documentation française, 1989 b.

Neyrand G., Savoirs et normes sociales en matière de petite enfance, Recherches et Prévisions, septembre-décembre $1999, n^{\circ} 57 / 58$.

Rack C., L'insertion professionnelle des jeunes filles, 4 Pages, CEE, novembre 1998, nº 30.

Silvera R., Les inégalités de salaire : rien de nouveau sous le soleil ?, Travail, automne-hiver 1994/1995, n 31/32.

Sineau M., Des femmes en politique, Paris, Economica, 1988.

Terrail J.-P., Destins scolaires de sexe, Population, 1992 a, n 3.

Terrail J.-P., Parents, filles et garçons, face à l'enjeu scolaire, Education et formation, 1992 b, n 30.

Terrail J.-P., Réusite scolaire : la mobilisation des fille, Sociétés contemporaines, 1992 c, n 11/12.

Terrail J.-P., Rapports de sexe et performances scolaire, Société française, hiver 1992/1993, nº 45.

Terrail J.-P., La dynamique des générations. Activité individuelle et changement social (1968/1993), Paris, L'Harmattan, 1995.

Travail, genre et société, "L'emploi est-il un droit», L'Harmattan, 1999, $\mathrm{n}^{\circ} 2$.

Zarca B., La division du travail domestique : poids du passé et tension au sein du couple, Economie et statistique, janvier 1990, $\mathrm{n}^{\circ} 228$. 